\title{
Quality Verification of Dendritic Cell-Based Cancer Vaccine
}

\author{
Shigetaka Shimodaira ${ }^{1 *}$, Terutsugu Koya1, Yumiko Higuchi ${ }^{1}$, Masato Okamoto ${ }^{2}$ and Shigeo Koido ${ }^{3}$
}

${ }^{1}$ Center for Advanced Cell Therapy, Shinshu University Hospital, Matsumoto, Nagano 390-8621, Japan

${ }^{2}$ Department of Advanced Immunotherapeutics, Kitasato University School of Pharmacy, Minato-ku 108-8641, Tokyo, Japan

${ }^{3}$ Department of Gastroenterology and Hepatology, The Jikei University School of Medicine, Kashiwa, Chiba 277-8567, Japan

\begin{abstract}
In the era of personalized cancer therapy, immunotherapy is now emerging as a potential option. Therapeutic cancer vaccination has been developed for the induction of an efficient immune response targeting tumor-associated antigens. The efficacy of dendritic cell (DC)-based vaccines is attributed to their ability to induce immunity against cancers. Criteria for the approval of therapeutically active DCs, such as their viability and purity, are here verified based on phenotypic characteristics, linked to their antigen-presenting ability and functional analyses of phagocytosis and pinocytosis. A standardized phenotype of DCs harboring bioactive functions would be useful to provide personalized vaccines for cancer immunotherapy.
\end{abstract}

Keywords: Dendritic cells, Cancer vaccination; Cytotoxic T cells; Phenotype; Antigen presentation; Phagocytosis; Pinocytosis

Abbreviations: DCs: Dendritic Cells; GM-CSF: GranulocyteMacrophage Colony-Stimulating Factor; IL: Interleukin; MDCs: Mature DCs; DCs: Immature; HLA: Human Leukocyte Antigen; WT1: Wilms' Tumor 1; CTLs: Cytotoxic T Cells; OK-432: Streptococcal Preparation; FITC: Fluorescein Isothiocyanate; PE: Phycoerythrin; MART-1: Melanoma Antigen Recognized by T Cells 1; PBLs: Peripheral Blood Lymphocytes; FCS: Fetal Calf Serum; MFIs: Mean Fluorescence Intensities

\section{Introduction}

Immunotherapy is now emerging as a potential option for personalized cancer therapy. Because of their specific bioactivity against tumor-associated antigens, vaccines using dendritic cells (DCs) may offer survival benefits in some patients with cancer by extending the number of quality-adjusted life years [1]. DCs with potential bioactivity used in active cancer immunotherapies [2-4] have been conventionally generated using peripheral monocytes with granulocyte-macrophage colony stimulating factor (GM-CSF) and interleukin (IL)-4 [5,6].

Mature dendritic cells (mDCs) express cell surface molecules necessary for antigen presentation. CD83, CD86, and human leukocyte antigen (HLA)-DR were found to stimulate $\mathrm{T}$ cells in vitro in almost all mDCs [7]. The expression of CD197 (C-C chemokine receptor type 7) was also shown to increase with maturation and induced chemotaxis associated with macrophage inflammatory protein $3 \beta$ [8]. It was also found that immature DCs (imDCs) have an endocytic pathway, such as antigen uptake through the mannose receptor and pinocytosis, which decreased after DC maturation [8].

DC vaccines primed with HLA class I/II-restricted Wilms' tumor 1 (WT1) peptides have been identified as a feasible option for patients with advanced cancers $[9,10]$. Immune response monitoring using tetramer analysis and/or enzyme-linked immunosorbent spot assay has been applied to determine the efficacy of DC vaccines targeting WT1 $[10,11]$. Because the standardized detection of cancer-associated antigens would also be useful for personalized cancer immunotherapy [12], clinical efficacy is also confirmed based on good targets in tumors for cancer vaccines that are effectively recognized by antigen-specific cytotoxic T cells (CTLs) [13]. These DC vaccines are required to meet the following criteria: purity defined as $>90 \%$ CD11c+ CD14-CD86+ HLA-DR+ cells, $>80 \%$ viability, mature DC phenotype, negative for bacterial and fungal infection after 14 days, presence of endotoxin $\leq 0.05 \mathrm{EU} / \mathrm{ml}$, and negative for mycoplasma [14]. In this article, we present our commentary on the findings obtained thus far and our insights regarding DC vaccines and vaccinations.

\section{Materials and Methods}

\section{A conventional IL-4-DC vaccine}

mDCs were generated under Good Gene, Cell \& Tissue Manufacturing Practice conditions according to "The Act on the Safety of Regenerative Medicine" introduced in Japan on November 25,2014 [15]. imDCs were generated by culturing adherent cells in AIM-V medium (Gibco, Gaithersburg, MD, USA) containing GMCSF (50 ng/ml; GENTAUR Belgium BVBA, Brussels, Belgium) and IL-4 (50 ng/ml; R\&D Systems Inc., Minneapolis, MN, USA) for 5 days using mononuclear cell-rich fractions isolated through apheresis, as previously described [14]. mDCs were differentiated from imDCs by stimulation with OK-432 $(10 \mu \mathrm{g} / \mathrm{ml}$ streptococcal preparation; Chugai Pharmaceutical Co., Ltd., Tokyo, Japan) and prostaglandin E2 (50 ng/ ml; Daiichi Fine Chemical Co., Ltd., Toyama, Japan) for $24 \mathrm{~h}$. mDC products were cryopreserved at $-152^{\circ} \mathrm{C}$ or in the gas layer of a liquid nitrogen tank until the day of administration.

\section{Phenotype of imDCs and mDCs}

The phenotype of mDCs was defined as CD11c+, CD14-, HLADR+, HLA-ABC+, CD80+, CD83+, CD86+, CD40+, and CD197+, as determined using flow cytometry [4]. DCs were stained using monoclonal antibodies conjugated with FITC or PE, such as CD14, CD40, CD83 (eBioscience, Inc., San Diego, CA, USA), HLA-ABC, CD80, CD86, CD11c (BD Biosciences, San Jose, CA, USA), and CD197 (R\&D Systems Inc.), for $30 \mathrm{~min}$ at $4^{\circ} \mathrm{C}$. CD3 and CD19 (BD Biosciences) were used for the detection of lymphocytes to evaluate DC purity. The expression of each surface marker on DCs was analyzed in

*Corresponding author: Shigetaka Shimodaira, Center for Advanced Cell Therapy, Shinshu University Hospital 3-1-1 Asahi, Matsumoto, Nagano 390-8621, Japan, Tel: +81-263-37-3580; Fax: +81-263-37-3027; E-mail: shimodai@shinshu-u.ac.jp

Received January 30, 2016; Accepted February 16, 2016; Published February 18,2016

Citation: Shimodaira S, Koya T, Higuchi Y, Okamoto M, Koido S (2016) Quality Verification of Dendritic Cell-Based Cancer Vaccine. Pharm Anal Acta 7: 465. doi:10.4172/2153-2435.1000465

Copyright: (c) 2016 Shimodaira S, et al. This is an open-access article distributed under the terms of the Creative Commons Attribution License, which permits unrestricted use, distribution, and reproduction in any medium, provided the original author and source are credited. 
the viable cells by excluding dead cells by processing with propidium iodide $(2 \mu \mathrm{g} / \mathrm{ml}$; Sigma-Aldrich Co. LLC., Tokyo, Japan). The phenotype was determined using flow cytometry (BD FACSCanto ${ }^{\mathrm{mt}} \mathrm{II}$; BD Biosciences).

\section{Functional analysis of DCs}

The antigen-presenting ability of both imDCs and mDCs in patients with HLA-A ${ }^{\star} 02: 01$ was studied using MART-1 [16-18]. In short, DCs were pulsed with $20 \mu \mathrm{g} / \mathrm{ml}$ melanoma antigen recognized by T cells 1 (MART-1)26-35 A27L (ELAGIGILTV) peptide at $37^{\circ} \mathrm{C}$ for $1 \mathrm{~h}$, followed by processing with mitomycin C (MMC, $25 \mu \mathrm{g} / \mathrm{ml}$; Kyowa Hakko Kogyo Co., Ltd., Tokyo, Japan) for 1 h. Peripheral blood lymphocytes (PBLs) as a responder were mixed with $1 \times 10^{6}$ of DCs as a stimulator at a ratio of 10:1, which were cultured in AIM-V medium containing IL-2 (Immunace, $2.5 \mathrm{U} / \mathrm{ml}$; Shionogi Pharmaceutical Co., Ltd., Osaka, Japan), IL-7 (5 ng/ml; R\&D Systems), and IL-15 (10 ng/ $\mathrm{ml}$; PeproTech, Rocky Hill, NJ, USA) for 3-5 days. Depending on the increase in PBLs, mixed cells were supplemented in AIM-V medium with $10 \%$ fetal calf serum (FCS), and additionally cultured for 2-3 days. Harvested cells were stained using CD8-FITC (Beckman Coulter, Inc., Brea, CA, USA), CD3-APC (eBioscience, Inc.), and T-select HLA-A *02:01 MART-1 tetramer-ELAGIGILTV-PE (MBL; Medical \& Biological Laboratories Co., Ltd., Nagoya, Japan) to detect MART-1specific CTLs. A negative control was applied as PBLs cultured without mixing with DCs.

The ability of DCs to perform phagocytosis and pinocytosis was evaluated in accordance with previous reports [19-21]. Both imDCs and $\mathrm{mDCs}$ were incubated with $10 \mu \mathrm{g} / \mathrm{ml}$ DQ-ovalbumin (Molecular Probes, Thermo Fisher Scientific K.K., Yokohama, Japan) for the evaluation of phagocytosis in AIM-V with 10\% FCS (Thermo Fisher Scientific K.K.) at $37^{\circ} \mathrm{C}$ for $30 \mathrm{~min}$. DCs incubated at $4^{\circ} \mathrm{C}$ were used as a negative control. The type of DC was determined using flow cytometry (BD FACSCanto ${ }^{\text {mex }} \mathrm{II}$ ).

A total of $1 \times 10^{6}$ cells $/ \mathrm{ml}$ of imDCs and mDCs were incubated with $250 \mu \mathrm{g} / \mathrm{ml}$ FITC-conjugated dextran (molecular weight, 40,000 Molecular Probes, Thermo Fisher Scientific K.K.) in AIM-V containing $10 \%$ FCS (Thermo Fisher Scientific K.K.) at $37^{\circ} \mathrm{C}$ for $3 \mathrm{~h}$ to clarify pinocytosis using flow cytometer (BD FACSCanto II). DCs reacted at $4^{\circ} \mathrm{C}$ were also analyzed as a negative control.

Wilcoxon signed rank test was applied to assess the differences between imDCs and mDCs. Univariate analysis of variance using the Dunnett's test was applied for multiple comparisons between PBLs cultured with imDCs or mDCs. A p value of $<0.05$ was set to indicate statistical significance using IBM SPSS Advanced Statistics ver. 23.0 (IBM Japan, Tokyo, Japan).

\section{Results}

\section{Phenotype of imDCs and mDCs}

Manufactured DCs primed with OK-432 were compatible with the mDC phenotype, expressing HLA- ABC+ DR+ CD40+ CD80+ CD86+ CD197+ as shown in Figure 1. mDCs were revealed to be more prominently consisting of a CD11c+/CD14-HLA-class I+/II+ population compared with imDCs. HLA-related molecules, such as CD80, CD83, and CD86, as well as CD40 and CD197, were expressed at higher levels in mDCs than in imDCs.

\section{Functional analysis of DCs}

The numerical value in the panel shows the ratio of induced MART1-specific CTLs (Figure 2A). Antigen-presenting ability was detected for both imDCs and mDCs, but it was confirmed that this ability was increased in mDCs, concomitant with their mature phenotype of HLAABC, HLA-DR, CD80, CD83, and CD86. The estimated marginal means $(\mathrm{N}=5)$ were $0.50,1.22$, and 4.32 in PBLs cultured without DCs, with imDCs, and mDCs, respectively. The mean different was significant between PBLs with mDCs and PBLs without DCs ( $\mathrm{p}=$ $0.001)$, and PBLs with imDCs $(\mathrm{p}=0.006)$, as shown in Figure $2 \mathrm{~B}$.

The ability of DCs to perform phagocytosis and pinocytosis was evaluated as shown in the left and right panels of Figure 2C, respectively. A solid line (blue, imDC; red, $\mathrm{mDC}$ ) indicates the reaction at $37^{\circ} \mathrm{C}$, and a dashed line indicates a negative control in Figure 2C. The median mean fluorescence intensities (MFIs) using DQ-ovalbumin to evaluate phagocytosis $(\mathrm{N}=9)$ were 1,882 and 1,436 in imDCs and mDCs, respectively, as shown in Table 1. The median MFIs using FITC-dextran to evaluate pinocytosis $(\mathrm{N}=9)$ were 462 and 239 in imDCs and mDCs, respectively. The manufactured $\mathrm{mDCs}$ shifted to lower intensities of phagocytosis ( $24 \%$ reduction in the median, $\mathrm{p}=0.015$ ) and pinocytosis ( $48 \%$ reduction in the median, $\mathrm{p}=0.038$ ) than the imDCs (Table 1 ).

\section{Discussion}

Despite recent advances in encouraging clinical results, a lot of elements of DC-based vaccine and vaccination strategies need to be optimized for successful implication for the cancer therapy [22,23]. The use of murine models can provide direct efforts toward an effective vaccine, and contribute to the design of successful DC-based cancer immunotherapy [24]. Anti-tumor immune response in the human setting was exploited with different DC-based vaccines to induce immune responses capable of controlling tumor growth and generating immune memory [25].

mDCs manufactured using GM-CSF and IL-4 primed with OK432 for clinical use expressed the HLA- ABC+ DR+ CD40+ CD80+ CD86+ CD197+ phenotype at a higher intensity that was sufficient for antigen presentation to detect MART-1-specific CTLs in in vitro model than imDCs, as shown in Figure 2. Authors conducted a phase I study to investigate the safety and immunogenicity using the DC vaccines targeting Wilms' tumor 1 manufactured using GM-CSF and IL-4 primed with OK-432 for patients with pancreatic cancer [9] and colorectal cancer [10]. The preclinical analyses on DC vaccines in vitro confirmed clinical efficacy to evaluate acquired cancer immunity based on immune monitoring using tetramer analyses and enzyme-linked immunosorbent spot assays.

On the other hand, mDCs shifted to lower levels of phagocytosis and pinocytosis than imDCs, as shown in Table 1. Efficacy of vaccines with mDCs for clinical use comprised activities of $76 \%$ phagocytosis and $52 \%$ pinocytosis. These profiles, other than antigen presentation, are labeled as functional tags in the preclinical and clinical development of DC-based anticancer therapeutics [26].

\section{Conclusion}

Qualification of the phenotype and functional analyses of the adapted DC vaccines would prove their efficacy for cancer immunotherapy.

\section{Disclosure of Interests}

All authors declare no conflicts of interest that could be perceived as prejudicing the impartiality of the reported research.

\section{Acknowledgement}

This study was supported by a Grant of Funding Programs: A-STEP (Adaptable and Seamless Technology Transfer Program through Target-driven R\&D) from the 
imDC
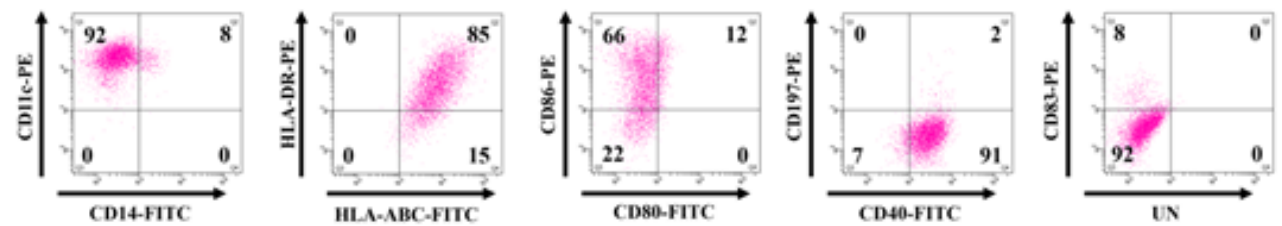

mDC
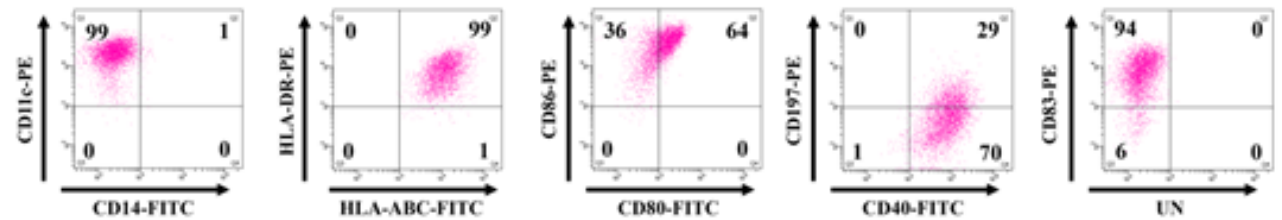

Figure 1: Phenotype analysis of immature DCs (imDCs) and mature dendritic cells (mDCs). The phenotype panels from patients with pancreatic cancer indicate $\mathrm{HLA}-\mathrm{ABC}{ }^{+} \mathrm{DR} \mathrm{R}^{+} \mathrm{CD} 40^{+} \mathrm{CD} 80^{+} \mathrm{CD} 86^{+} \mathrm{CD} 197^{+}$, which should meet the criteria. Upper panels are for imDCs and lower ones for mDCs. The numbers in quadrants indicate the percentage of the panels.

(A)

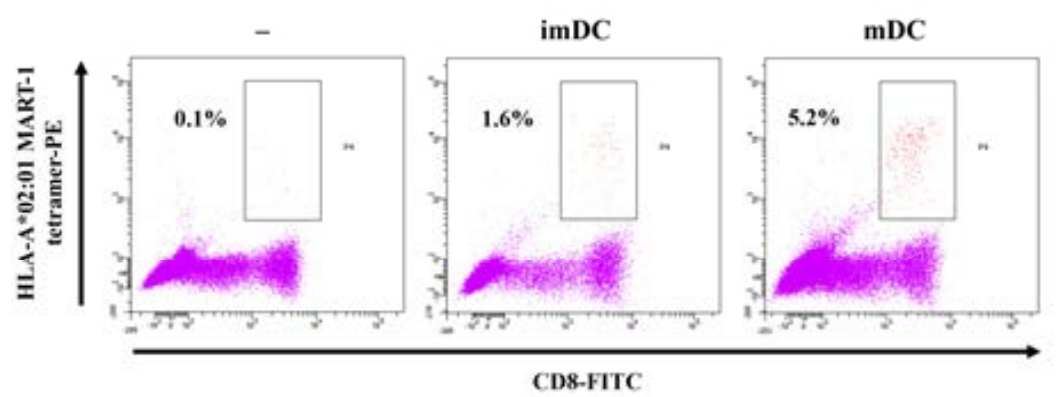

(B)

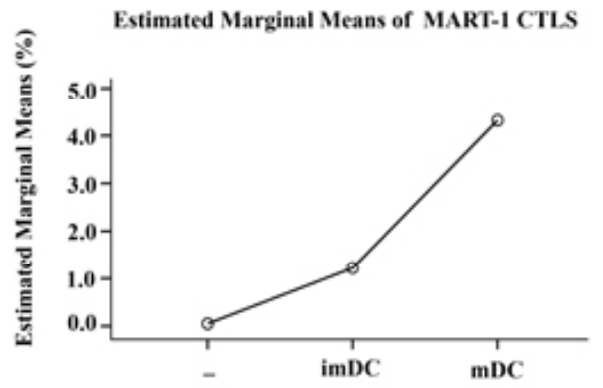

(C)

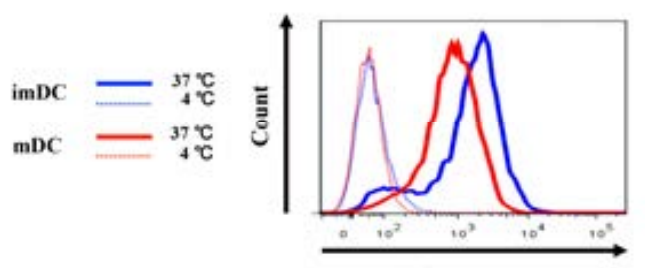

DQ-ovalbumin

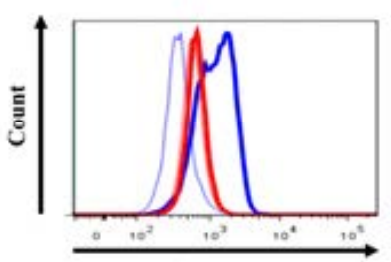

FITC-dextran

(A) Manufactured mDCs applied for clinical use exhibited increased antigen presentation using the MART-1 system. (B) Estimated marginal means of MART-1 specific CTLs. *, $p<0.05$ indicates statistical significance using Dunnett's test. (C) The manufactured mDCs (red lines) shifted to lower levels of phagocytosis (DQovalbumin) and pinocytosis (FITC-dextran) compared with the imDCs (blue lines).

Figure 2: Antigen presentation, phagocytosis and pinocytosis of imDCs and $\mathrm{mDCs}$. 
Japan Society and Technology Agency. The authors would like to thank Enago (www.enago.jp) for the English language review.

\section{Grants} Agency.

The study was supported by a grant from the Japan Society and Technology

\section{References}

1. Shimodaira S, Higuchi Y, Yanagisawa R, Okamoto M, Koido S (2016) Efficacy of dendritic cell-based cancer immunotherapy. Biochem Anal Biochem.

2. Banchereau J, Steinman RM (1998) Dendritic cells and the control of immunity Nature 392: 245-252.

3. Steinman RM, Banchereau J (2007) Taking dendritic cells into medicine. Nature 449: 419-426.

4. Figdor CG, de Vries IJ, Lesterhuis WJ, Melief CJ (2004) Dendritic cell immunotherapy: mapping the way. Nat Med 10: 475-480.

5. O'Neill DW, Adams S, Bhardwaj N (2004) Manipulating dendritic cell biology for the active immunotherapy of cancer. Blood 104: 2235-2246.

6. Nestle FO, Farkas A, Conrad C (2005) Dendritic-cell-based therapeutic vaccination against cancer. Curr Opin Immunol 17: 163-169.

7. Sato K, Nagayama H, Tadokoro K, Juji T, Takahashi TA (1999) Extracellular signal-regulated kinase, stress-activated protein kinase/c-Jun $\mathrm{N}$-terminal kinase, and p38mapk are involved in IL-10-mediated selective repression of TNF-alpha-induced activation and maturation of human peripheral blood monocyte-derived dendritic cells. J Immunol 162: 3865-3872.

8. Sato K, Kawasaki H, Nagayama H, Enomoto M, Morimoto C, et al. (2000) TGFbeta 1 reciprocally controls chemotaxis of human peripheral blood monocytederived dendritic cells via chemokine receptors. J Immunol 164: 2285-2295.

9. Koido S, Homma S, Okamoto M, Takakura K, Mori M, et al. (2014) Treatment with chemotherapy and dendritic cells pulsed with multiple Wilms' tumor 1 (WT1)-specific MHC class I/II-restricted epitopes for pancreatic cancer. Clin Cancer Res 20: 4228-4239.

10. Shimodaira S, Sano K, Hirabayashi K, Koya T, Higuchi Y, et al. (2015) Dendritic cell-based adjuvant vaccination targeting Wilms' tumor1 in patients with advanced colorectal cancer. Vaccines 3: 1004-1018.

11. Higuchi Y, Koya T, Yuzawa M, Yamaoka N, Mizuno Y, et al. (2015) Enzymelinked immunosorbent spot assay for the detection of Wilms' tumor1-specific $T$ cells induced by dendritic cell vaccination. Biomedicines 3: 304-315.

12. Shimodaira S, Sano K, Hirabayashi K, Koya T, Higuchi Y, et al. (2015) Dendritic Cell-Based Adjuvant Vaccination Targeting Wilms' Tumor 1 in Patients with Advanced Colorectal Cancer. Vaccines (Basel) 3: 1004-1018.
13. Everson RG, Antonios JP, Lisiero DN, Soto H, Scharnweber R, et al. (2015) Efficacy of systemic adoptive transfer immunotherapy targeting NY-ESO-1 for glioblastoma. Neuro Oncol.

14. Shimodaira S, Higuchi Y, Koya T, Kobayashi T, Yanagisawa R, et al. (2015) Smoking influences the yield of dendritic cells for cancer immunotherapy. Pharmaceut Reg Affairs 4: 1.

15. Shimodaira S, Hirabayashi K, Kobayashi T, Higuchi Y, Yokokawa K (2015) Future prospective of cancer vaccination technology in Japan. Pharmaceut Reg Affairs 4: 2

16. Sasawatari S, Tadaki T, Isogai M, Takahara M, Nieda M, et al. (2006) Efficient priming and expansion of antigen-specific CD8+ T cells by a novel cell-based artificial APC. Immunol Cell Biol 84: 512-521.

17. Akiyama Y, Maruyama K, Tai S, Takikawa M, Ohshita C, et al. (2008) Novel approach to the characterization of melanoma associated-peptide-specific CTL lines from Japanese metastatic melanoma patients. Int J Oncol 33: 433-441.

18. Muto M, Baghdadi M, Maekawa R, Wada H, Seino K. (2015) Myeloid molecular characteristics of human $y \delta T$ cells support their acquisition of tumor antigenpresenting capacity. Cancer Immunol Immunother 64: 941-949.

19. Daro E, Pulendran B, Brasel K, Teepe M, Pettit D, et al. (2000) Polyethylene glycol-modified GM-CSF expands CD11b (high) CD11c (high) but notCD11b(low) CD11c(high) murine dendritic cells in vivo: a comparative analysis with Flt3 ligand. J Immunol 165: 49-58.

20. Sixt M, Kanazawa N, Selg M, Samson T, Roos G, et al. (2005) The conduit system transports soluble antigens from the afferent lymph to resident dendritic cells in the T cell area of the lymph node. Immunity 22: 19-29.

21. Lapenta C, Santini SM, Spada M, Donati S, Urbani F, et al. (2006) IFN-alphaconditioned dendritic cells are highly efficient in inducing cross-priming CD8(+) T cells against exogenous viral antigens. Eur J Immunol 36: 2046-2060.

22. Obeid J, Hu Y, Slingluff CL Jr (2015) Vaccines, Adjuvants, and Dendritic Cell Activators--Current Status and Future Challenges. Semin Oncol 42: 549-561.

23. Mody N, Dubey S, Sharma R, Agrawal U, Vyas SP (2015) Dendritic cell-based vaccine research against cancer. Expert Rev Clin Immunol 11: 213-232.

24. Mac Keon S, Ruiz MS, Gazzaniga S, Wainstok R (2015) Dendritic cell-based vaccination in cancer: therapeutic implications emerging from murine models. Front Immunol 6: 243.

25. Pizzurro GA, Barrio MM (2015) Dendritic cell-based vaccine efficacy: aiming for hot spots. Front Immunol 6: 91.

26. Bloy N, Pol J, Aranda F, Eggermont A, Cremer I, et al. (2014) Trial watch: Dendritic cell-based anticancer therapy. Oncoimmunology 3: e963424. 